\title{
Scanner path planning with the control of overlap for part inspection with an industrial robot
}

\author{
Nguyen Duy Minh Phan • Yann Quinsat • \\ Sylvain Lavernhe • Claire Lartigue
}

Received: date / Accepted: date

\begin{abstract}
Automated inspection of manufactured parts is increasingly getting attention as it helps to make a rapid decision on product conformity. In this context, the aim of this paper is to present a new scanner path planning method for part inspection using an industrial 6-axis robot The novelty of the approach is to generate a scan path with the control of the overlap between 2 adjacent scanning paths based on the use of the least-squares conformal maps, which stretches a 3D mesh surface on a $2 \mathrm{D}$ plane. Equidistant paths calculated in the $2 \mathrm{D}$ space are then transformed into equidistant paths in the 3D space. The effective performance of controlling the overlap can improve digitizing quality and save digitizing time by managing the coverage of the laser beam. Furthermore, the digitizing quality is also ensured by keeping a constant scanning distance and executing a continuous control of the scanner orientation relatively to the part surface for all the driven points of the scan path. An experimental application of this new approach is proposed for a laser-plane scanner mounted on an industrial robot with 6 degrees of freedom, which demonstrates the interest of such an approach.
\end{abstract}

Keywords Automated inspection · Robot · Laser-Scanner trajectory · Overlap control

M. Phan

LURPA, ENS Paris-Saclay, Univ. Paris-Sud, Université Paris-Saclay, 94235 Cachan, France

Tel.: +33147402213

Fax: +33147402220

E-mail: nguyen-duy-minh.phan@ens-paris-saclay.fr

Y. Quinsat

E-mail: yann.quinsat@ens-paris-saclay.fr

S. Lavernhe

E-mail: sylvain.lavernhe@ens-paris-saclay.fr

C. Lartique

E-mail: claire.lartigue@ens-paris-saclay.fr 


\section{Introduction}

Inspection procedures increasingly rely on the use of laser-plane sensors due to their great ability to measure part defects within a minimum amount of time. When mounted on industrial robots, sensors' accessibility is largely increased; this gives the sensor the possibility to scan an object from any direction even along curved paths [1]. Within this context, scan path planning remains a major challenge to obtain a complete representation of the surface in a minimum amount of time with a given scanning quality. Scan path can be defined as a set of scanner configurations (or view points), i.e. a set of driven points and associated orientations (Figure 1). These configurations are classically obtained by imposing some constraints to the sensor in relation to the part to be measured. In the literature, visibility and quality are the most usual constraints. Initially dedicated to sensors mounted on Coordinate Measuring Machines (CMM), methods have evolved in the last decade to adapt to robots equipped with optical sensors. For robots, additional degrees of freedom give the sensor greater accessibility to the surfaces to be measured. It is thus possible to generate more complex and continuous scan paths, e.g. trajectories that leave the sensor orientation continually perpendicular to the surface. Additional constraints, such as the control of scanning overlap can thus be integrated in the trajectory calculation. Indeed, scanning overlap, if uncontrolled, may affect both measuring time and measuring quality. Within this context, we propose a method of scan path planning with the control of overlap. The originality lies in the use of a conformal-map based method, which is usually rather used in tool path planning for machining, or in gun path planning for painting. Here, the difficulty resides in taking into account laser-scanner specificities. The paper is organized as follows. Section 2 is dedicated to related works. Section details our approach which is then illustrated in section 4 through an example.
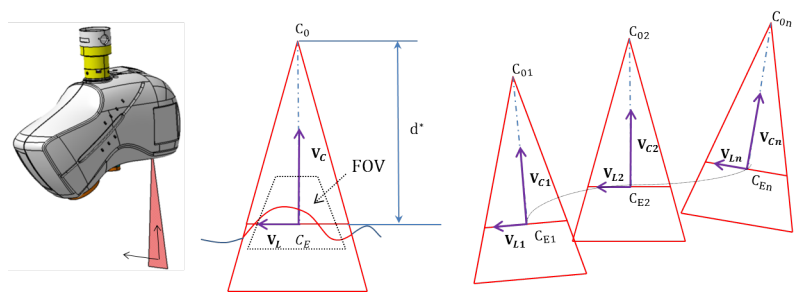

Fig. 1 Laser-scanner trajectory and parameters

\section{Related works}

\subsection{Scan path planning}

Determining view points based on the concept of visibility consists in finding the surface portions that belong to the field of view (FOV) of the scanner. The FOV is the part of the laser beam which is visible by the scanner camera ([11]). Considering 
the laser-scanner mounted on a CMM, Bernard and Véron [2] introduce three levels of visibility (local, global and real) to generate a sensor trajectory welladapted to the control of complex parts. Their approach relies on the unitary sphere or Gaussian sphere, which is tesselated using a STL format. The part is also tessellated, and the local visibility is obtained when the angle between the normal vector to the part triangle and the normal vector to a triangle of the unitary sphere is lesser than a specific threshold. The global visibility is computed by finding visibility directions that intersect with other triangles of the part. Finally, real visibility is obtained after achieving the collision test between the part and the sensor. From the CAD model, $\mathrm{Xi}$ and $\mathrm{Shu}$ determine the optimal parameters of the scanner FOV to maximize the portion of scanned surface [3]. The surface is divided into sections by cutting the CAD model using parallel cross-sections. For each section, the optimal position is obtained by aligning the top of the FOV with the upper boundary of the surface profile. While ElMaraghy and Yang [4] have developed an approach based on the surface decomposition into several patches. For each patch, a zigzag trajectory is defined in function of the viewing angle and the depth of view (DOV). Then the total path is calculated by aggregating all the trajectories. Derigent et al. propose to use the notion of global and local visibility through $2 \mathrm{D}$ visibility maps [5]. The local visibility cone (LVC) of a face F consists of the set of view directions for which the angle between the view direction and the vector normal to $\mathrm{F}$ is lesser than the maximum view angle. The set of view directions that intersect with the part defines the occulatation cone (OC), and finally, the boolean subtraction between LVC and OC gives the global visibility cone. Lee and Park [6] define the locally accessible directions (LADs) at each point of the surface mesh by respecting some constraints such as the view angle, the depth of the view, the check of collisions with the part or the scanner. The sets of globally accessible directions (GAD) are obtained by performing Boolean intersection operations among LADs.

In addition to visibility, some studies propose to define points of view according to quality criteria. Prieto et al. [7] propose to keep the scanner normal to the surface, while obeying a quality criterion depending on both the scanning distance and the sensor view angle. Mahmud et al. [9] build the scan path by limiting the number of orientations of the laser-scanner, and considering an optimal digitizing distance defined as the middle of the scanner FOV. Yang and Ciarallo use a genetic algorithm to obtain a set of viewing domains and a list of observable entities for which the errors are within an admissible tolerance [10]. The approach developed by Lartigue et al. [11] relies on the representation of the part surface as a voxel map, for which the size of each voxel is defined according to the size of the scanner FOV. To each voxel, a unique point of view is associated according to visibility and quality criteria. Those criteria are defined by admissible ranges of digitizing distances and view angles so as to ensure the surface digitizing with a given quality. Martin et al. propose a method based on voxelization of the CAD model which takes into account various criteria: surface coverage, scanning quality and scan path length [12]. The volume of the 3D box which surrounds the surface is divided into slices. The slice width is given by the laser stripe, and for each slice, a collision free scan path is generated. Consecutive paths are combined together according to a zig-zag strategy to define the final trajectory. Besides visibility and quality constraints, Son et al. include additional constraints such as the number of required scans and the checking of occlusions [13]. Mavrinac et al. [14] formalize the search of 
sensor viewpoints under constraints for 3D inspection using an active triangulation system. Each viewpoint is assessed thanks to a performance function that results from the combination of constraint functions to be respected.

Raffaeli et al. [15] propose a view planning approach for an optical 3D scanner mounted on robot arms. Their approach relies on a NURBS surface which is divided into portions that are included in the scanner field of view, and for which the variations of the normal vectors do not exceed an angular limit. The points that can be seen from the same point of view are gathered according two conditions: the maximum distance between two points in the same cluster must be less than the scanner field of view, and the maximum angle between the normal vectors must be less than $90^{\circ}$. Wu et al. [16] propose a method of sensor path planning for surface inspection on a 6 degree-of-freedom robot that automatically adapts its trajectory to the complex shape of the object by continuously changing the viewing direction of the scanner mounted on the robot. Each viewpoint of the planned path must however satisfy several constraints: field of view, scanning distance, view angle and overlap. First, a wireframe representation of the CAD model is extracted as the projection contour of the model in the main direction, which is computed as the mean of all the normal vectors in the model. Next, the model is divided into several digitizing regions using the rectangle whose dimensions are defined by the FOV of the scanner and the overlap constraint. Koutecky et al. [17] have recently described a method for a ATOS system mounted on a KUKA robot. The surface is divided into cubes, according to the FOV and to the scanner depth of view. Their approach consists in finding the orientations of the system in order to have the greatest number of visible polygonal facets as possible. Larsson and Kjellander propose an approach for scan path planning using a laser-scanner mounted on a robot dedicated to unknown objects [1]. A first scan is made from four orthogonal directions; the scanner is positioned at the limits of the working space of the robot. From data acquired during this first scan, a shape scan step is performed to retrieve the approximate shape of the object. Mineo et al.[18] develop a MATLAB toolbox named RoboNDT in order to generate the raster scan paths for surface inspection by the 6-axis industrial robots. Starting from the triangular mesh imported from the CAD model, the idea is to generate a raster scan path defined as a succession of curves and associated normal vectors. A curve, parallel to one edge of the surface, at a distance d from the edge, is calculated as the intersection of a plane and the meshed surface. Each point of the obtained curve lays on one of the triangles of the mesh, and the perpendicular direction associated to each point is thus given by the normal vector of the relative triangle. The process is iterated to generate equally spaced parallel lines.

Most of the methods previously detailed described the scan path as a discrete set of points of view, which are defined according to quality and visibility constraints. Only a few studies address the issue of scanning overlap between two successive passes. The overlap zone represents the surface portion covered by two consecutive passes (Figure 2), and may alter scanning time and quality.

\subsection{Scanning overlap Control}

To demonstrate the influence of scanning overlap on quality, a reference plane is scanned according to a zig-zag strategy for which the percentage of overlap is fixed 


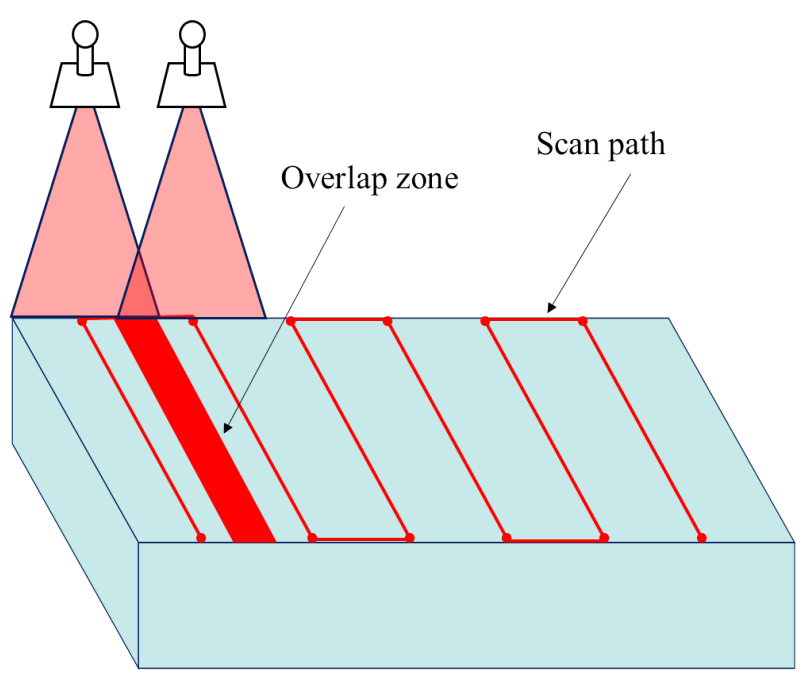

Fig. 2 Definition of the overlap zone

to 25 of the laser-line width. Two different types of overlap are considered. The first overlap zone (overlap 1 in Figure 2) results from two consecutive passes with the same scanner orientation. For the second overlap zone (overlap 2 in Figure 2), two sensor orientations are used. Digitizing quality can be evaluated in terms of digitizing noise. To evaluate the digitizing noise, digitized point clouds are registered to the nominal surface. For this purpose, the CAD model of the reference plane is meshed. For each facet of the reference mesh, a cylinder is created, whose basis is the triangle defining the facet. The set of digitized points belonging to the cylinder so defined corresponds to the actual digitized facet. For each facet, the distances between the digitized points and the facet are calculated. The associated standard deviation represents the actual digitizing noise. The evolution of digitizing noise over all the surface is shown in the Figure 3(a). The point density for the overlap zones is always higher than in other zones and as a result, digitizing noise in overlap zones is greater than in non-overlap zones. This difference is particularly significant in the overlap zone 2 for which the sensor orientation has been modified. Figure 3(b) clearly highlights the superposition effect of the two point clouds leading to a larger dispersion and, as a result, a higher value of noise. As the scanning is performed using a robot, the laser beam can be continuously reoriented during the measurement which is a source of possible non-quality. Moreover, Figure 3(b) shows a little trueness error. As a result, the control of scanning overlap becomes necessary to control the scanning quality.

Overlapping control has been more studied in relation with gun-path planning for painting with an industrial robot. Indeed, in such cases, the overlap control is essential to obtain the desired paint thickness and uniformity. In order to minimize time cycles as well as to control paint thickness uniformity, painting path strategies designed to control painting overlap have been proposed in some studies. In their approach, Andulkar et al. [19] calculated the optimal overlap distance between two consecutive passes according to the distribution model of the paint. In [20], 


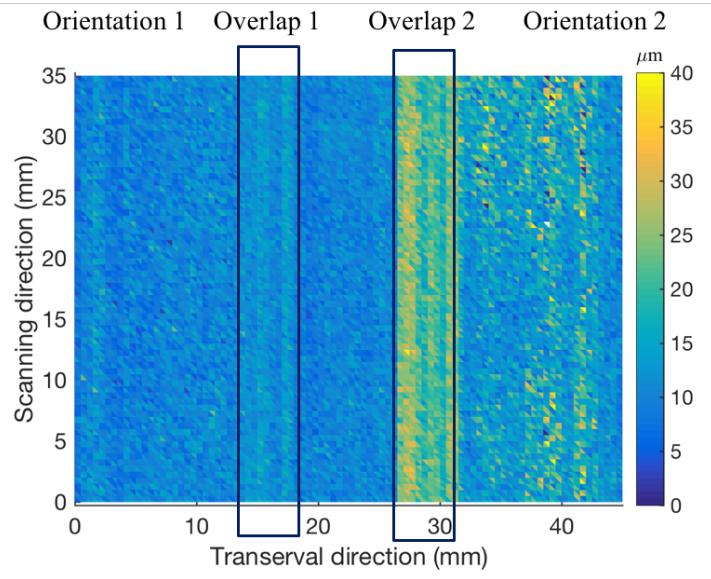

(a) Digitizing noise evaluated on the reference plane

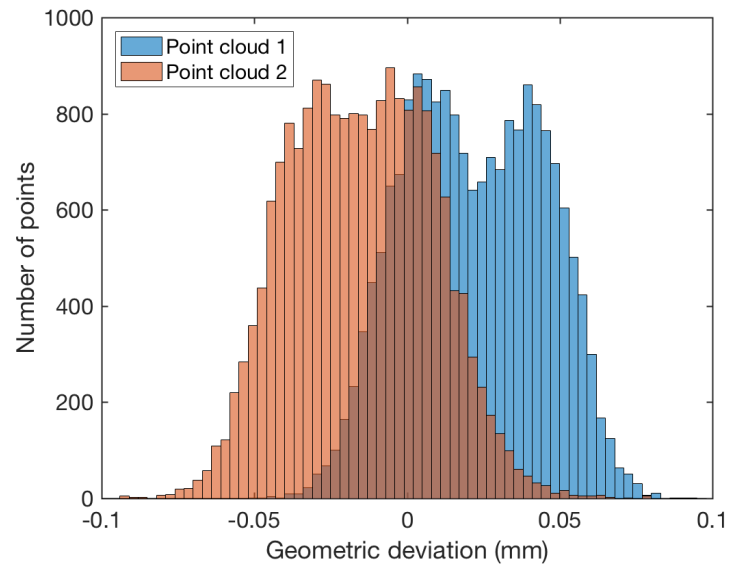

(b) Histogram of the geometrical deviations in zone 2

Fig. 3 Influence of scanning overlap on digitizing noise

the authors show that the generation of a spray gun trajectory that uniformly covers the surface not only relies on the definition of the path orientations and the spacing between passes but also on the speed along the passes.

Controlling the overlap between two successive scanning passes also presents similarities with constant scallop-height tool path methods for milling. The isoscallop tool path strategy can be used in machining to improve the machined surface quality and to minimize the machining time by scheduling the cutter paths so that the scallop height left by the tool between two successive paths is constant. Tournier and Duc [21] proposed a method based on the machining surface concept $M S$, which is the offset surface by $R$ of the nominal surface, with $R$ the tool radius. Each pass of the trajectory $C_{i}$ belongs to $M S$. The envelope surface of the tool movement associated to $C_{i}$ defines a pipe surface $P_{i}$ whose radius is equal to 
$R$. Authors define the iso-scallop height surface $S S h$ which is the offset surface by $S h$, the expected scallop height, of the nominal surface. Then, to ensure a constant scallop height, two adjacent tool paths $C_{i}$ and $C_{i+1}$ are built so that the intersection curve (scallop curve) between the two pipe surfaces $P_{i}$ and $P_{i+1}$ belongs to the iso-scallop-height surface $S S h$. Can and Unuvar [22] developed an iso-scallop tool path generation algorithm which calculates the iso-scallop cutter contact points from the analysis of the B-spline surface. The cutter tool radius is adjusted to the surface curvature, and then side step is computed for a constant scallop height. A surface curvature analysis procedure is applied to the entire surface to determine the minimum radius of curvature along the feed and the sidestep directions. This gives the maximum allowable radius of a tool to be used for machining the given surface.

Some works propose an interesting approach based on the conformal map [23] [24]. The 3D mesh of the surface is stretched onto a $2 \mathrm{D}$ plane using the conformal map. The advantage of the conformal map is to locally preserve the shape: the distances and the areas are only changed by a scaling factor [25]. Then, equidistant passes calculated in the $2 \mathrm{D}$ space can be transformed into iso-scallop paths in $3 \mathrm{D}$ space by the inverse conformal map. The great advantage here is the simplification of calculation to control the overlap, as the tool path generation is performed in the $2 \mathrm{D}$ space. In this paper, we propose an original approach to generate a scan path planning for a laser-plane scanner mounted on an industrial robot that takes advantage of the conformal map to control the overlap between two adjacent scanning passes. A scanning path with the control of digitizing overlap allows the management of both the orientation and the coverage rate of the laser beam.

\section{Iso-Overlap Scan-path method - ISOvScan}

In our approach, the digitizing system consists of a laser-plane sensor mounted on an industrial robot. The sensor trajectory is defined as a set of ordered scanner configurations, i.e. a set of positions $C_{E}$ and orientations $\left(\mathbf{V}_{\mathbf{L}} ; \mathbf{V}_{\mathbf{C}}\right)$, with $\mathbf{V}_{\mathbf{L}}$ the director vector of the digitizing line, and $\mathbf{V}_{\mathbf{C}}$ the director vector of the light-beam axis. The point $C_{E}$ positions the scanning laser line in the field of view: $\mathbf{C}_{\mathbf{0}} \mathbf{C}_{\mathbf{E}}=$ $d^{*} \cdot \mathbf{V}_{\mathbf{C}}$, with $d^{*}$ the scanning distance (Figure 1). The proposed method to control digitizing overlap is inspired by iso-scallop tool path methods more commonly used for machining. To simplify computation, the scanner trajectory is calculated in the $2 \mathrm{D}$ space of the part surface, as proposed in [23]. Nevertheless, as the CAD model of the surface is not a continuous surface model but a 3D mesh, it is necessary to transform the 3D mesh surface into a 2D parametric space, using the Least-Square Conformal Map method (LSCM). The method we developed, called ISOvScan for Iso-Overlap Scan path, presents three main steps:

- stretching the 3D mesh surface on a $2 \mathrm{D}$ parametric surface using the LSCM method

- generating the view points $c_{e}$ in the $2 \mathrm{D}$ space, and transformation of $c_{e}$ into $C_{E}$ in the $3 \mathrm{D}$ space by the inverse LSCM

- calculation of the scanner orientations for each view point according to quality constraints

Each step of the ISOvScan method is detailed next. 
Table $13 \mathrm{D}$ and 2D parameters

\begin{tabular}{ll|ll}
\hline & $3 D$ mesh parameters & & 2D mesh parameters \\
\hline$S_{T}$ & set of $n$ triangular facets & $s_{t}$ & set of $n$ triangular facets \\
$T_{j}$ & facet $j$ with $T_{j} \in S_{T}, j \in[1, n]$ & $t_{j}$ & facet $j$ with $t_{j} \in s_{t}, j \in[1, n]$ \\
$S_{v}$ & set of vertices in 3D & $s_{v}$ & set of vertices in 2D \\
$V_{j}^{k}$ & one vertex of the facet $T_{j}$ & $v_{j}^{k}$ & one vertex of the facet $t_{j}$ \\
$\left(x_{j}^{k}, y_{j}^{k}\right)$ & with $k \in[1,3]$ & & with $k \in[1,3]$ \\
$A_{T_{j}}$ & coordinates of the vertex $V_{j}^{k}$ & $\left(u_{j}^{k}, v_{j}^{k}\right)$ & coordinates of the vertex $v_{j}^{k}$ \\
$\mathbf{n}_{\mathbf{j}}$ & normal vector to the facet $T_{j}$ & $a_{t_{j}}$ & area of the facet $t_{j}$ \\
\hline
\end{tabular}

3.1 Mapping between the 3D space and the 2D parametric space using LSCM

First, let us introduce the LSCM method. In complex analysis, a conformal map is defined as a bijection that locally keeps the angles [26]. The transformation of a $3 \mathrm{D}$ triangulated surface into a $2 \mathrm{D}$ space $(u, v)$ can be considered as a complex function:

$$
\psi(s)=u(x, y)+i v(x, y)
$$

with $s=x+i y$, and $(x, y)$ the coordinates in the local basis (Figure 4 ).

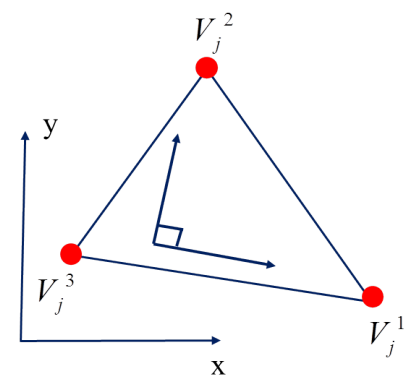

Fig. 4 A triangle provided with a local basis

A function is said to be conformal if it satisfies the Cauchy-Riemann conditions:

$$
\left\{\begin{array}{l}
\frac{\partial u}{\partial x}=\frac{\partial v}{\partial y} \\
\frac{\partial u}{\partial y}=-\frac{\partial v}{\partial x}
\end{array}\right.
$$

which can also be written as [27]:

$$
\nabla v=\left(\begin{array}{cc}
0 & -1 \\
1 & 0
\end{array}\right) \nabla u
$$

Considering notations defined in table 1 , the facet $T_{j}$, of $S_{T}$ of the surface is provided with the orthonormal basis $(x, y)$ (Figure 4 ). 
In this basis, the function which maps a point $(x, y)$ of the triangle to a point $(u, v)$ in the parametric space yields:

$$
\left\{\begin{array}{l}
u(x, y)=\lambda_{1} \cdot u_{j}^{1}+\lambda_{2} \cdot u_{j}^{2}+\lambda_{3} \cdot u_{j}^{3} \\
v(x, y)=\lambda_{1} \cdot v_{j}^{1}+\lambda_{2} \cdot v_{j}^{2}+\lambda_{3} \cdot v_{j}^{3}
\end{array}\right.
$$

where $\lambda_{1}, \lambda_{2}$, and $\lambda_{3}$ denote the barycentric coordinates of the point $(x, y)$, calculated as follows:

$$
\left(\begin{array}{c}
\lambda_{1} \\
\lambda_{2} \\
\lambda_{3}
\end{array}\right)=\frac{1}{2 \cdot A_{j}} \cdot\left(\begin{array}{c}
y_{j}^{2}-y_{j}^{3} x_{j}^{3}-x_{j}^{2} y_{j}^{3} x_{j}^{2}-y_{j}^{2} x_{j}^{3} \\
y_{j}^{3}-y_{j}^{1} x_{j}^{1}-x_{j}^{3} y_{j}^{1} x_{j}^{3}-y_{j}^{3} x_{j}^{1} \\
y_{j}^{1}-y_{j}^{2} x_{j}^{2}-x_{j}^{1} y_{j}^{2} x_{j}^{1}-y_{j}^{1} x_{j}^{2}
\end{array}\right) \cdot\left(\begin{array}{c}
x \\
y \\
1
\end{array}\right)
$$

Then, $\nabla u$ can be written as a function of the barycentric coordinates:

$$
\nabla u=\left(\begin{array}{c}
\frac{\partial u}{\partial x} \\
\frac{\partial u}{\partial y}
\end{array}\right)=M_{T_{j}} \cdot\left(\begin{array}{c}
u_{j}^{1} \\
u_{j}^{2} \\
u_{j}^{3}
\end{array}\right)
$$

with:

$$
M_{T_{j}}=\frac{1}{2 \cdot A_{j}} \cdot\left(\begin{array}{ccc}
y_{j}^{2}-y_{j}^{3} & y_{j}^{3}-y_{j}^{1} & y_{j}^{1}-y_{j}^{2} \\
x_{j}^{3}-x_{j}^{2} & x_{j}^{1}-x_{j}^{3} & x_{j}^{2}-x_{j}^{1}
\end{array}\right)
$$

The condition of equation 3 leads to:

$$
M_{T_{j}} \cdot\left(\begin{array}{c}
v_{j}^{1} \\
v_{j}^{2} \\
v_{j}^{3}
\end{array}\right)-\left(\begin{array}{cc}
0 & -1 \\
1 & 0
\end{array}\right) \cdot M_{T_{j}}\left(\begin{array}{l}
u_{j}^{1} \\
u_{j}^{2} \\
u_{j}^{3}
\end{array}\right)=\left(\begin{array}{l}
0 \\
0
\end{array}\right)
$$

As equation 8 can only be respected for developable surfaces, for general surfaces the equation is verified in the least-squares sense. Let us denote $E_{L S C M}$ nonconformality. The least-squares method consists in searching the set of parameters $\left(u_{j}^{k}, v_{j}^{k}\right)$ which minimize $E_{L S C M}$ :

$$
E_{L S C M}=\sum_{T_{j} \in S_{T}} A_{T_{j}} \cdot\left\|M_{T_{j}} \cdot\left(\begin{array}{c}
v_{j}^{1} \\
v_{j}^{2} \\
v_{j}^{3}
\end{array}\right)-\left(\begin{array}{cc}
0 & -1 \\
1 & 0
\end{array}\right) \cdot M_{T_{j}}\left(\begin{array}{l}
u_{j}^{1} \\
u_{j}^{2} \\
u_{j}^{3}
\end{array}\right)\right\|^{2}
$$

After the implementation of the LSCM algorithm, a facet $T_{j}$ of the $3 \mathrm{D}$ surface is transformed into the facet $t_{j}$ in the $2 \mathrm{D}$ parametric space, and the set $S_{T}$ of $\mathrm{n}$ triangular facets in the $3 \mathrm{D}$ space is then transformed into the set $s_{t}$ of $\mathrm{n}$ triangular facets in the 2D space (Figure 5).

The next step is the generation of the view points, or driven points, $c_{e}$ in the $2 \mathrm{D}$ space, followed by the transformation of $c_{e}$ into $C_{E}$ in the $3 \mathrm{D}$ space by the inverse LSCM. 


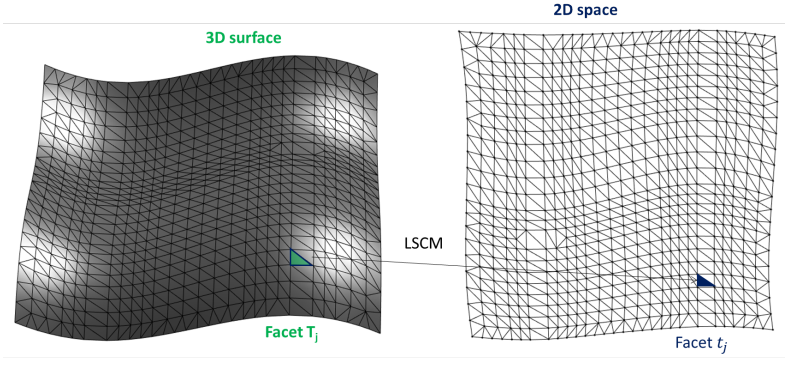

Fig. 5 A 3D surface transformed into a 2D surface by LSCM

\subsection{Generation of the driven points}

The scan path is generated in 2D considering a classical parallel-plane trajectory. The length of the rectangle that bounds the 2D surface defines the plane direction, and the driven points are determined by the intersection between parallel planes and the parametric surface. To control the overlap, parallel planes are equidistant, with $I_{2 D}$ the distance between two successive parallel planes corresponding to the distance between two successive passes in the 2D space. However, it is the distance $I_{3 D}$ between two successive passes in the $3 \mathrm{D}$ space that controls the overlap. Therefore, a relationship between $I_{2 D}$ and $I_{3 D}$ is established considering that the ratio of both values is equal to the proportionality coefficient of similar triangles $T_{j}$ and $t_{j}$ :

$$
\frac{I_{3 D}}{I_{2 D}}=\frac{1}{n} \sum_{j=1}^{n} \sqrt{\frac{A_{T_{j}}}{a_{t_{j}}}}
$$

The coordinates of the driven points $c_{e i}\left(c_{e 1}, c_{e 2},, c_{e 3}\right)$ in the $2 \mathrm{D}$ space are thus obtained. A driven point $c_{e i}$ is transformed into its corresponding point $C_{E i}$ by the inverse transformation (Figure 6).
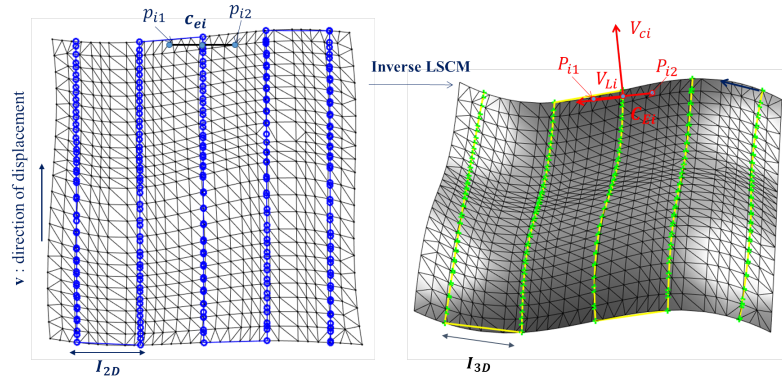

Fig. 6 Calculation of the scanning trajectory

Once all driven points are calculated, the next step is to determine the sensor orientations for each driven point. This is performed in two steps: first the director 
vector of the digitizing line $\mathbf{V}_{\mathbf{L}}$ is determined, before the light-beam axis $\mathbf{V}_{\mathbf{C}}$ is calculated.

\subsection{Determination of the director vector}

In order to achieve good scanning quality for the entire surface, the digitizing distance must belong to a range of admissible values, generally determined from the scanner assessment [28]. A constant scanning distance is first imposed to all the driven points to ensure that the digitized surface at the driven point is visible in the FOV of the scanner, and also to ensure an expected digitizing quality. The width of the laser line, denoted $L_{o p t}$, associated to this digitizing distance, is constant with respect to the scanned surface. In order to maximize the digitized surface, the laser line must be perpendicular to the direction of displacement along the scanner trajectory in the 3D space. Then, the laser line must also be perpendicular to the direction of displacement of the trajectory in the $2 \mathrm{D}$ space, denoted $v$ (figure $6)$. The laser-line width $l_{o p t}$ in the $2 \mathrm{D}$ space is defined using the proportionality coefficient: $l_{o p t}=L_{o p t} \cdot I_{2 D} / I_{3 D}$. At each point $c_{e i}$, the laser-line is positioned perpendicularly to the path and centered at $c_{e i}$. The width $l_{\text {opt }}$ defines the two end points $p_{i 1}$ and $p_{i 2}$ of the laser line (Figure 6). The coordinates of the corresponding points $P_{i 1}$ and $P_{i 2}$ are calculated using the LSCM inverse transformation. Thus, the director vector of the digitizing line at driven point $C_{E i}$ is given by:

$$
\mathbf{V}_{\mathbf{L i}}=\frac{\mathbf{P}_{\mathbf{i} 1} \mathbf{P}_{\mathbf{i} 2}}{\left\|\mathbf{P}_{\mathbf{i} 1} \mathbf{P}_{\mathbf{i} 2}\right\|}
$$

Note that, such a mode of calculation imposes the digitizing distance to be fixed to a predefined value (the value leading to $L_{\text {opt }}$ ).

\subsection{Determination of the light beam axis}

The vector of light-beam axis $\mathbf{V}_{\mathbf{C}}$ is determined so that the scanning direction is always perpendicular to the surface. We thus have to calculate the local normal vector to the surface at each driven point $C_{E i}$. In this direction, the normal vector is calculated as the mean value of all the normal vectors to the facets associated to the laser line at $C_{E i}$. It is worth noting that due to the LSCM transformation, the facets associated to the laser line in the 3D space are also the facets associated to the laser line in the $2 \mathrm{D}$ space. These facets intersect with the laser line in the $2 \mathrm{D}$ space, and intersection points are easy to calculate (Figure 7). Therefore, after the transformation in the $3 \mathrm{D}$ space, the local normal vector is given by:

$$
\mathbf{n}_{\mathbf{C}_{\mathbf{E} \mathbf{i}}}=\frac{\sum_{j=1}^{m i} \frac{\mathbf{n}_{\mathbf{j}}}{A_{T_{j}}}}{\left\|\sum_{j=1}^{m i} \frac{\mathbf{n}_{\mathbf{j}}}{A_{T_{j}}}\right\|}
$$

where $m i$ is the number of facets related to the laser line at point $C_{E i}$, and $\mathbf{n}_{\mathbf{j}}$, the normal vector to the facet $T_{j}$ with $(j=1$ to $m i)$. Finally, the director vector of light-beam axis is defined at each driven point $C_{E i}$ by: $\mathbf{V}_{\mathbf{C i}}=\mathbf{n}_{\mathbf{C}_{\mathbf{E i}}}$.

The whole trajectory is thus obtained as a set of positions and orientations $\left(C_{E i}\right.$ $\left.; \mathbf{V}_{\mathbf{L i}} ; \mathbf{V}_{\mathbf{C i}}\right)$. The scan path planning method with overlap control ISOvScan is 


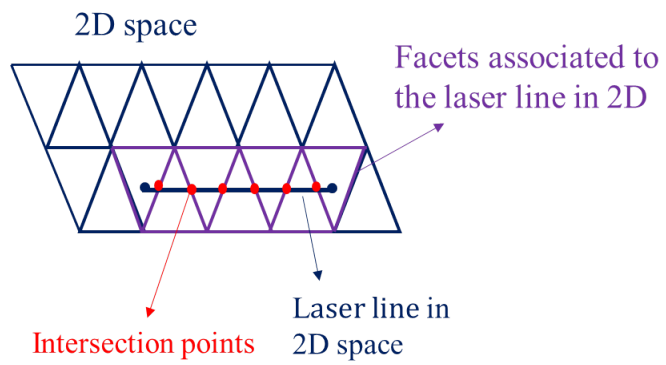

Fig. 7 Identification of the facets associated to the laser line

implemented in Matlab(c). By modifying the value of the pass interval $I_{3 D}$, the overlap zone can be controlled. Two examples are proposed in Figure 8.

\subsection{Illustration on an industrial case}

In order to illustrate the contribution of our approach, this section is dedicated to the comparison of the ISOvScan method with a classical zig-zag method. The chosen surface is a part of a ski mask mold (Figure 9).

Two different methods are used to compute the scan path. On the one hand, the ISOvScan method is used. Considering $L_{o} p t=18 \mathrm{~mm}$, the value $14.5 \mathrm{~mm}$ is chosen for $I_{3 D}$, corresponding to an overlap of $20 \%$. On the other hand, the scan path is generated by a zig-zag parallel plane strategy with a constant transversal step. For this case, the scanner orientation is constant for all the paths. In order to be able to compare both methods, the transversal step is computed considering the angular variations between the local vector and the laser beam axis all surface long. The mean angle directly computed form the CAD model is equal to $48^{\circ}$. Therefore, the transversal step is equal to $I_{3 D} * \cos \left(48^{\circ}\right)$, which guarantees that the local mean value of the distance between paths on the $3 \mathrm{D}$ surface (as defined in [8]) is equal to $I_{3 D}$. Paths generated by both methods are displayed in the Figures $10(\mathrm{a})$ and $10(\mathrm{~b})$.

Both scan paths have almost an equivalent length: the zig-zag trajectory is approximately $6 \%$ longer. To enhance the relevancy of our method, the simulation of the scanning is carried out using a simulator previously developed [29]. The scanning simulator relies on the analysis of the scanner configurations relatively to the meshed part(Figures 11(a) and 11(b)). Green facets correspond to well digitized facets using only one configuration, yellow facets belong to overlap zones, whereas red areas correspond to not digitized facets. As expected, the ISOvScan method (Figure 11(a)) has almost constant overlapping areas. The surface is also completely digitized. Concerning the zig-zag trajectory (Figure 11(b)), as the transversal step is constant, this implies that overlapping zones considerably vary. Minimal in the center of the part (where the slope is small), the width of the overlapping zones is much larger on part sides. This suggests that the quality of the digitizing will be poorly controlled in these areas. Moreover, due to the constant orientation of the scanner, the flanks of the part with a deep slope are not 


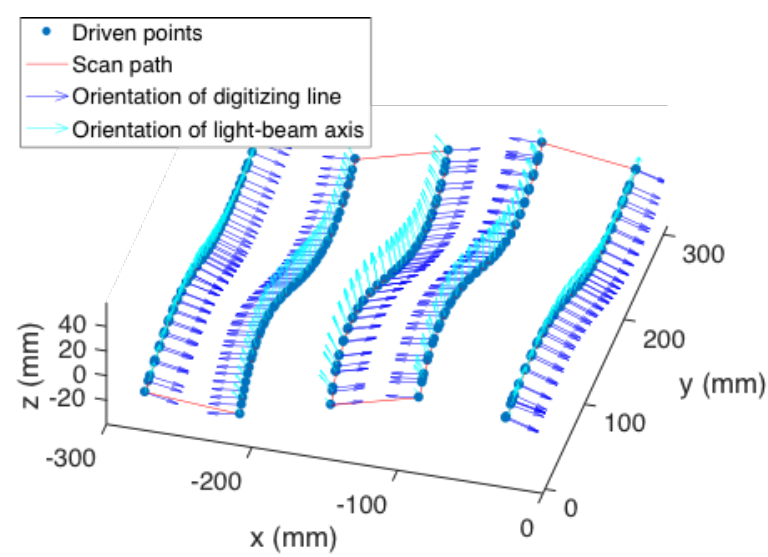

(a) Pass interval $I_{3 D}=65 \mathrm{~mm}$

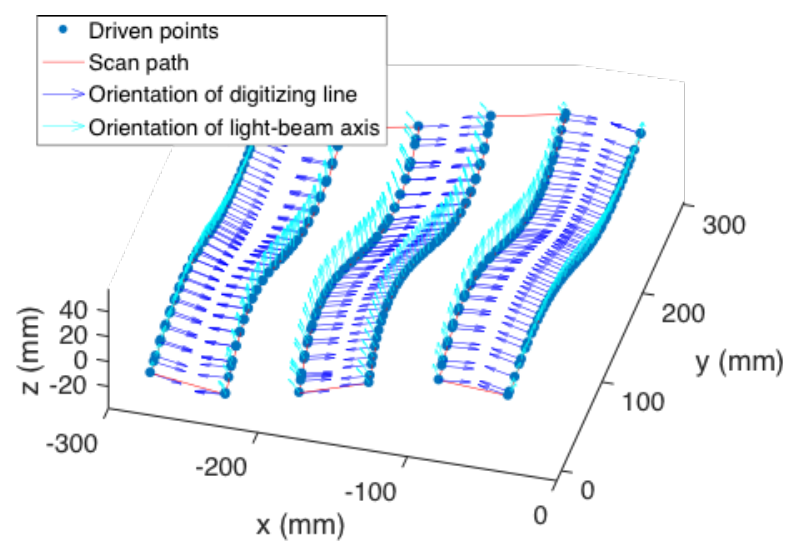

(b) Pass interval $I_{3 D}=50 \mathrm{~mm}$

Fig. 8 Examples of scanning trajectories for different pass intervals

digitized. This example clearly illustrates the interest of our approach to generate scan path with the control of quality, and the completeness of the measured data.

\section{Application and experimentation using a test surface}

In order to assess the ISOvScan method, an application is proposed using a scanning system which consists of a laser-plan sensor Zephyr II (www.kreon3d.fr), mounted on an industrial Mitsubishi 6-axis robot and an optical tracker (Figure 12(a)). To avoid the deviations of the effective laser position from the nominal one caused by the path execution, this system provides an independent measurement of the scanner positions. Indeed, the optical tracking device can track the positions of the scanner and the scanner's movements are then directly synchronized with the measurement. The proposed test part for this application is represented in Figure 12(b). 


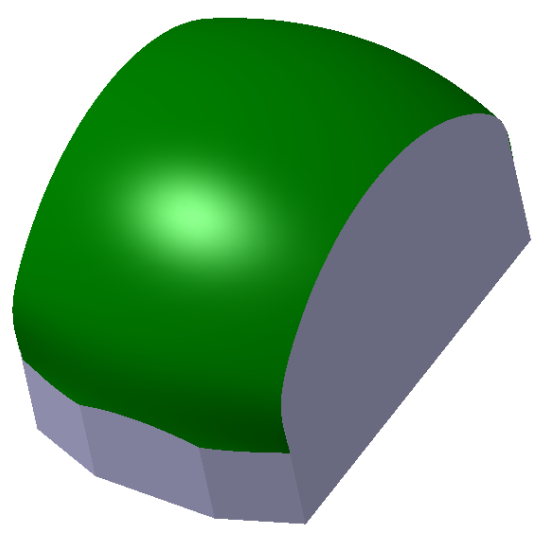

Fig. 9 Representation of the tudied surface

The ISOvScan method requires some scanner parameters, such as the dimensions of the FOV, and the admissible range of scanning distances which determine scanning quality. Therefore, a protocol of scanner assessment is applied to identify the actual scanner parameters.

\subsection{Scanner parameters}

As previously mentioned, the method requires a good knowledge of the scanner FOV. More precisely, we need to evaluate the range of digitizing distances allowing a good scanning quality. This is performed using the protocol QUALIPSO, detailed in [28], by measuring a reference plane. Considering the laser-line as the intersection between the reference plane and the laser-beam, the depth $H$, which corresponds to the height of the FOV, is the height for which the laser-line is visible in the CCD (Figure 13). The FOV is represented in red dotted-lines in the figure. The value of $H$ given by the experiment is $115 \mathrm{~mm}$. The digitizing noise is determined as the standard deviation between the measured points and the associated Least-Square plane. The evolution of the noise according to the digitizing distance displayed in Figure 13 is obtained by changing the scanning distance every $15 \mathrm{~mm}$ from the top to the bottom of the FOV. The figure exhibits a significant increase of the noise at the bottom position and the top position compared with the middle position. The admissible range of scanning distance is established considering a threshold for the noise. For our experiment, we set this threshold to $0.015 \mathrm{~mm}$, giving the admissible range of distances [35 $\mathrm{mm} ; 79 \mathrm{~mm}$ ]. If the surface scanned belongs to this range, this ensures a scanning with a noise lesser than $\delta_{a d}=0.015 \mathrm{~mm}$. Furthermore, the figure points out that the minimum value of noise corresponds to the digitizing distance $d^{*}=60 \mathrm{~mm}$ from the top of the FOV, giving the laser-line width $L_{o p t}=77 \mathrm{~mm}$. This digitizing distance is therefore adopted for the experiment. 


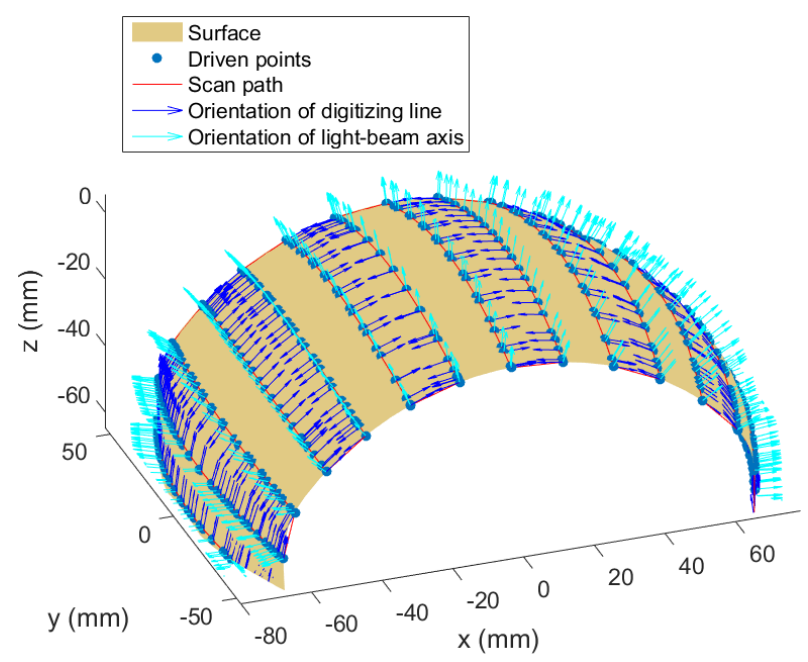

(a) ISOvScan scan path

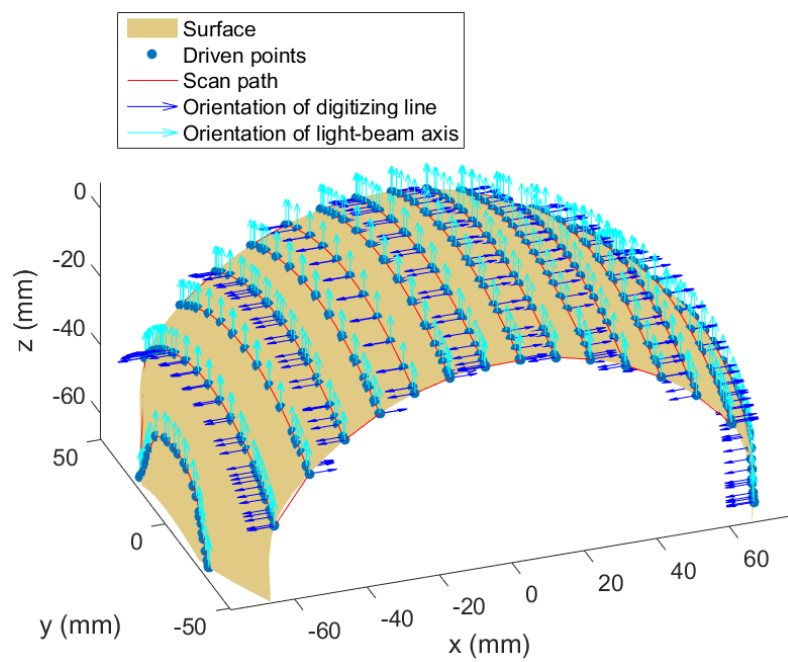

(b) Zigzag scan path

Fig. 10 Scan path representation

The choice of the overlap value must result from a compromise between the number of paths and the scanning noise. Experiments, performed by measuring a reference plane with various percentages of overlap, have highlighted that the percentage of the overlap should be lesser than $25 \%$ to optimize the number of paths. Experiments also reveal that the noise strongly increases when overlap is greater than $15 \%$. Therefore, the selected value for the overlap is $15 \%$, which imposes $I_{3 D}=65 \mathrm{~mm}$ considering $L_{o p t}=77 \mathrm{~mm}$. Due to the uncertainties in the 


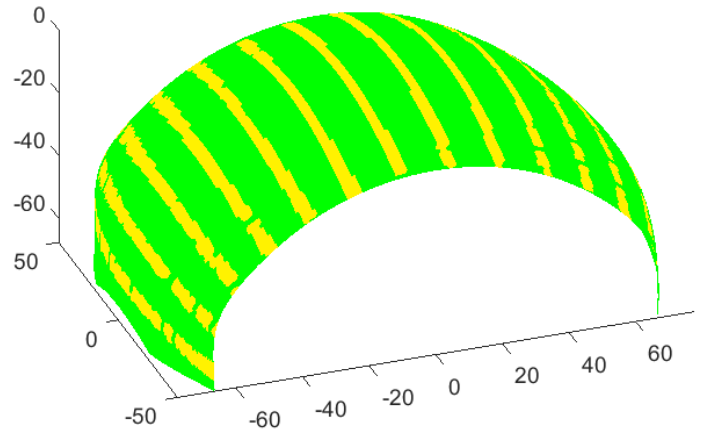

(a) ISOvScan simulation

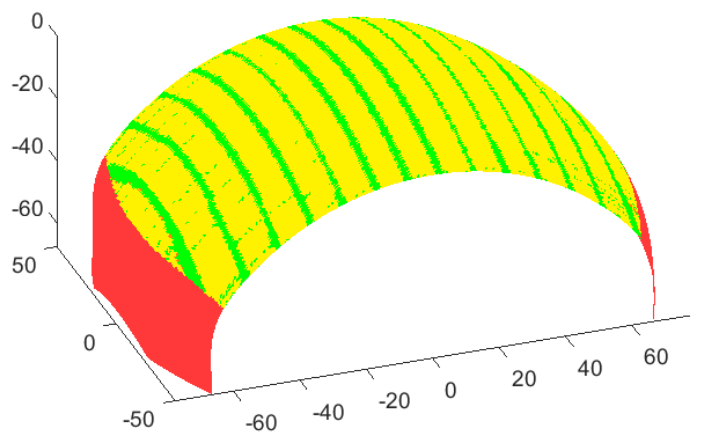

(b) Zigzag simulation

Fig. 11 Scanning simulation representation

modelling of the FOV and in the position of the driven point, a value too small could lead to unmeasured areas which is not desirable.

\subsection{Experiments}

The proposed method is applied to the part defined in Figure 12(b) using the parameters defined in section 4.1. The calculated scan path is reported in Figure 14(a). This scan path is first assessed by the scanning simulator previously developed [29]. The scanning simulation shows an expected digitizing result in which the most facets on the surface are digitized with a good digitizing quality (in green) and the dimension of the overlap zones between the successive passes are nearly constant (in yellow).

The scan path so calculated is thus transmitted to the industrial robot which supports the scanner to be executed. Prior to the execution, the robot's movements are simulated using RoboDK(C)(Figure 14(a)). The simulation gives the relative positions and orientations of firstly, the surface to be scanned and the robot, and secondly the laser sensor and the 6th robot axis. The study of the robot joint sollicitations assess the consistency of the trajectory in the robot coordinate system. The robot is equipped with an optical tracker system which acquires the 


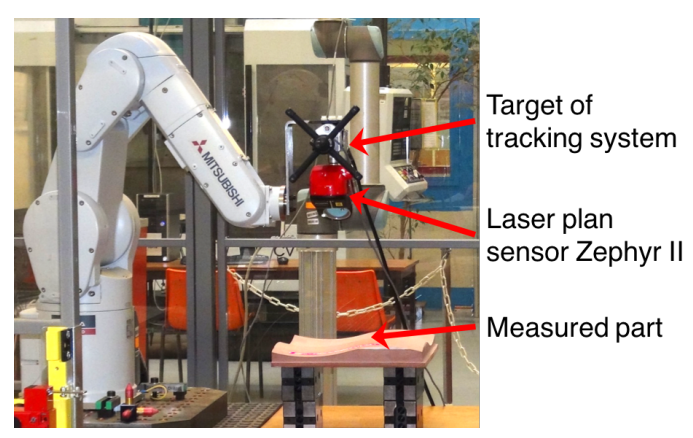

(a) Scanning system

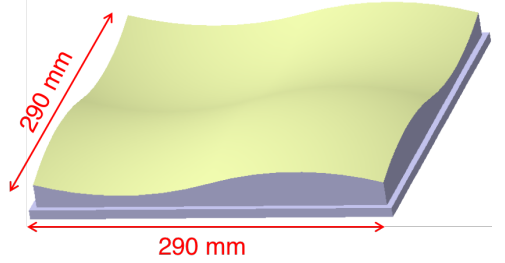

(b) CAD model of the test part

Fig. 12 Description of the studied application

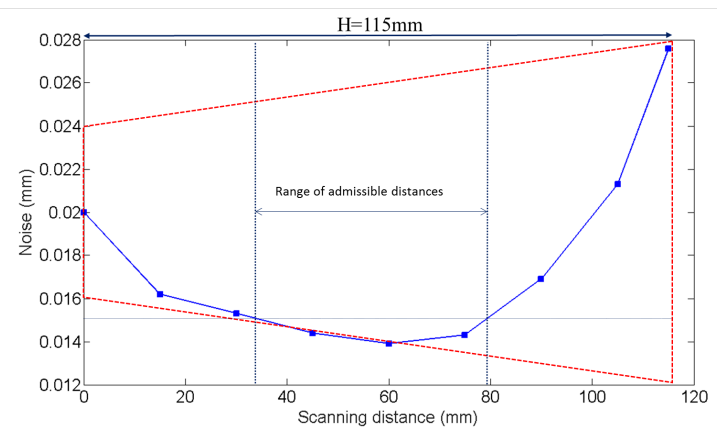

Fig. 13 Scanning noise in function of the scanning distance

scanners positions in an independent frame, whereas the point cloud is acquired using Kreon Polygonia software.

4.3 Results and discussions

The scanning gives a set of $1,629,604$ points. After the point cloud registration on the CAD model, a cartography of geometrical deviations is obtained (Figure 15). Both steps (registration and geometrical deviation calculation) are performed using the open source software CloudCompare (www.danielgm.net/cc/). The cartography reported in Figure 15 clearly highlights that the main deviations are 


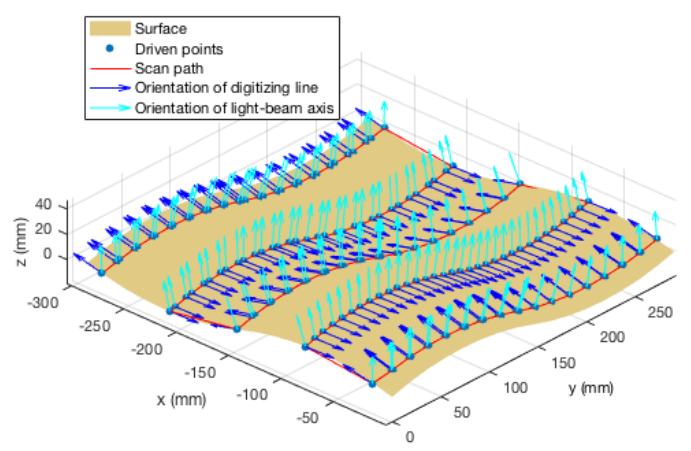

(a) Scan path generated by ISOvScan

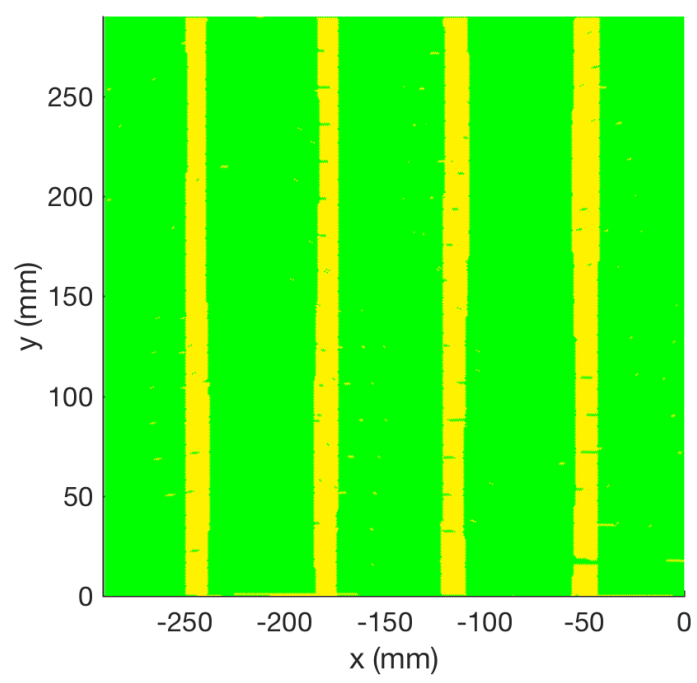

(b) Overlap simulation (Yellow) [29]

Fig. 14 Calculated scan path and scanning simulation

located at one border of the surface, which is likely due to the measuring conditions. Some scanning holes are also present on the point cloud due to the instability of laser beam during the scanning process. Since the driven points in 2D space are generated from the intersection between the equidistant planes and the facets, the scan path is a set of driven points, some of which are closely spaced, others not so close. Therefore, there is sudden change of scanner orientation when the scanner moves from one point to another. This may cause undesirable noise in some areas on the surface.

The evolution of the actual scanning noise is reported in Figure 16(a), in which the scale of colors accounts for the scanning noise evolution. As expected, results 


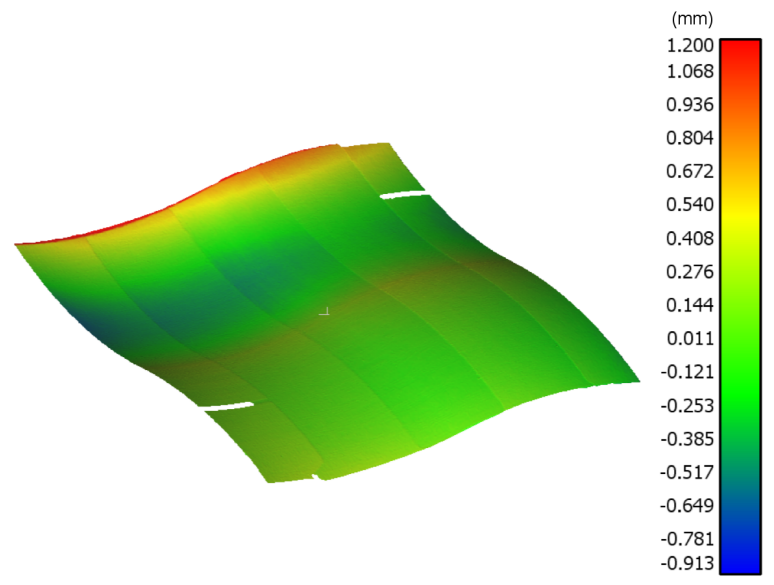

Fig. 15 Geometrical deviations between the point cloud and its CAD model

demonstrate that the digitizing noise in the overlap zones (yellow zones) is higher than for non-overlap zones.

Moreover, since we considered that the value of noise $0.015 \mathrm{~mm}$ was a threshold for quality, actual scanned data can also be assessed in terms of quality (Figure $16(\mathrm{~b}))$. If the noise is greater than the threshold $\delta_{a d}=0.015 \mathrm{~mm}$, the facet is labeled as obtained with poor quality, and its color is set to orange. Conversely, if the noise is lesser than $\delta_{a d}$, the facet is labeled as scanned with a good quality, and its color is green. As expected, orange facets, obtained by low-quality scanning, are generally located in overlap zones. Actual results shown in Figure 16(a) exhibit a close similarity with results obtained by simulation in Figure 14(b). Overlap zones are clearly zones of poor scanning quality.

Interestingly, the scanning noise is not uniform for all the overlapping zones. For instance, the scanning noise in zone (4) is the highest. As previously presented, it is very likely that the orientation changes between consecutive passes have led to different scanning noises for the different overlapping zones. To provide an explanation of this issue, we propose to analyze the geometrical deviation distribution in the overlap zones. The point cloud acquired in an overlap zone results from the combination of two point clouds acquired by two successive scan paths. Zone (2) and zone (4) in Figure 16(a), which corresponds to the lowest-noise and the highest-noise overlap zones, are specifically analyzed.

Results displayed in Figure 17(a) bring out that the dispersion of the geometrical deviations in the overlap zone (2) is smaller than the one in the overlap zone (4) in Figure 17(b). The analysis confirms the impact of overlap zone on the digitizing quality when scanner orientations vary. The noise in the overlap zone (2) is $\delta=0.030 \mathrm{~mm}$, where it equals $\delta=0.061 \mathrm{~mm}$ in zone (4). Different phenomena may explain this increase in the dispersion. The first one is linked to tracking error of the scanner when it moves from a position to another one. The second one can be related to the position of the measured surface within the FOV. Indeed, although the digitizing distance is constant, the visible portion is not necessarily located at the middle of the FOV, and can be at the border of the 


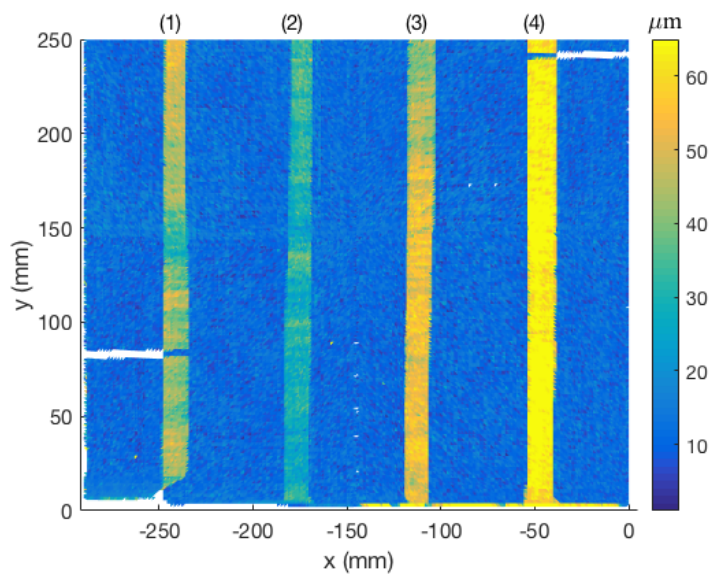

(a) Scanning noise evolution on the surface

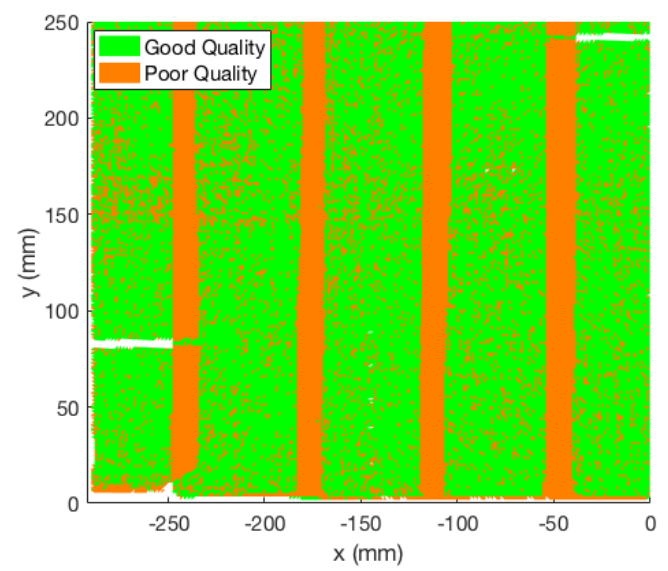

(b) Categorization of scanning noise in terms of quality

Fig. 16 Scanning noise and quality

FOV which leads to a higher dispersion. Finally, as already discussed, variations of orientations clearly affect the scanning quality. This result emphasizes how important the overlap control in scan path generation is. Indeed, the scanning noise in overlap zones is always greater than in non-overlap zones. The scanning quality and scanning time can be controlled by keeping a constant scanning distance and executing a continuous control of the scanner orientation for all the driven points of the scanning path. However, this experiment assesses the efficiency of the ISOvScan method for scan path planning with the control of overlap. 


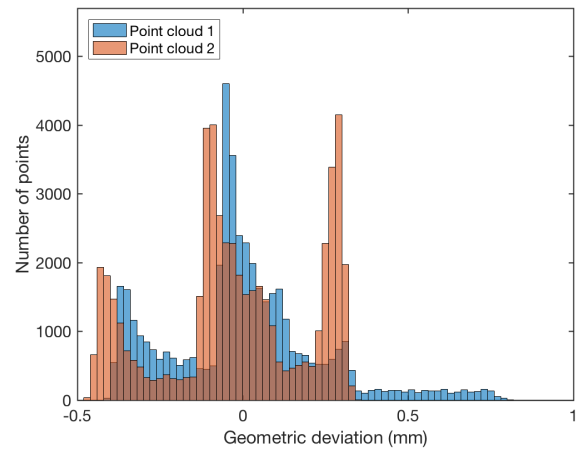

(a) Overlap zone (2)

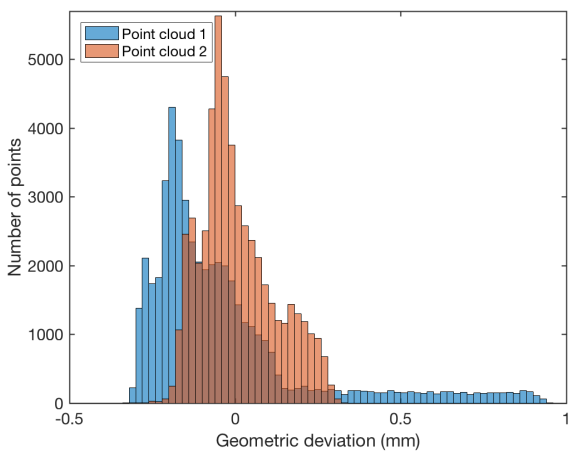

(b) Overlap zone (4)

Fig. 17 Scanning quality evaluation in overlap zones

\subsection{Limitations}

One limitation of the ISOvScan method is that the interval between the passes of the scan path in 3D space cannot be kept constant for all the scan path for high-curvature surfaces. In this case, the size of overlapping zones between passes is not uniform. This is due to errors made in the approximation of the conformal transformation. Actually, the interval between two successive passes is established according to Equation 10. The transformation from 3D space to 2D space is carried out for each facet of the surface, whereas the ratio $\frac{I_{3 D}}{I_{2 D}}$ is calculated as a mean value of the proportionality coefficient of similar triangles $T_{j}$ and $t_{j}$. Due to the approximation performed by LSCM, some errors exist in particular concerning the proportionality coefficient. Let us denote $k_{j}$, the LSCM error which represents the difference between the proportionality coefficient of two similar triangles $T_{j}$ and $t_{j}$ and the ratio $\frac{I_{3 D}}{I_{2 D}} \cdot k_{j}$ is given by:

$$
e_{L S C M}^{j}=\left|\frac{k_{j}-\bar{k}}{\bar{k}}\right|
$$


where

$$
\left\{\begin{aligned}
k_{j} & =\sqrt{\frac{A_{T_{j}}}{a_{t_{j}}}} \\
\bar{k} & =\frac{I_{3 D}}{I_{2 D}}=\frac{1}{n} \sum_{j=1}^{n} \sqrt{\frac{A_{T_{j}}}{a_{t_{j}}}}
\end{aligned}\right.
$$

For the whole meshed surface:

$$
\overline{e_{L S C M}}=\frac{1}{n} \sum_{j=1}^{n} e_{L S C M}^{j}
$$

The LSCM error is calculated for two examples of surfaces with different level of curvature variations. With a low-curvature variation surface, the interval between two consecutive passes is almost the same for all the passes (Figure 18). In the case of a high-curvature variation surface (Figure 19) the interval cannot be maintained uniformly in some zones on the surface. Obviously, the LSCM error corresponding to the high-curvature surface $(3.14 \%)$ is higher than the one for the low-curvature surface $(1.04 \%)$. It is thus possible to predict if the method will provide equally overlap zones or not by evaluating the LSCM error. Despite its limitation in the case of high-curvature surfaces, the ISOvScan can control the overlapping zones in many cases by combining accordingly the interval between passes and the laser width of the scanner. Nevertheless, it is worth noting that our method is more efficient when the wavelength of the geometrical defects is greater than the laser width. If not, our positioning method becomes non-consistent, and may cause visibility problems or may hinder overlap control as the visibility is only analysed for the driven points, and not for the whole laser line. In further research, we suggest that an alternative method should be proposed, where the sensor orientation could not be defined as continuously perpendicular to the surface.

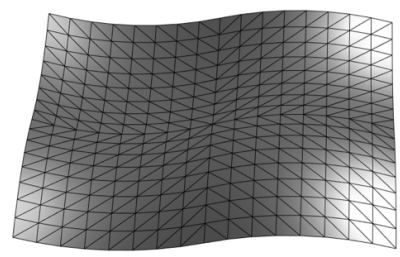

(a) Surface representation

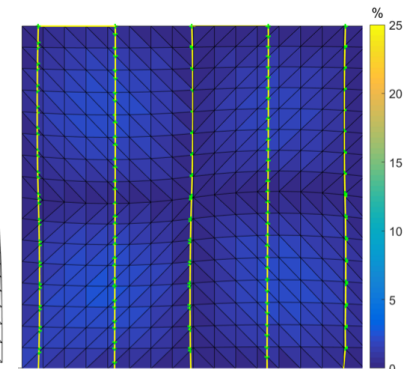

(b) Evolution of LSCM error (c) Overlap $\overline{e_{L S C M}}=1.04 \%$

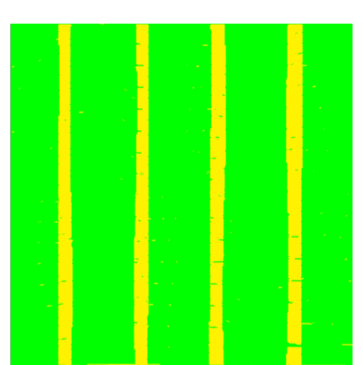

(Yellow)

Fig. 18 LSCM error for a low-curvature variation surface 


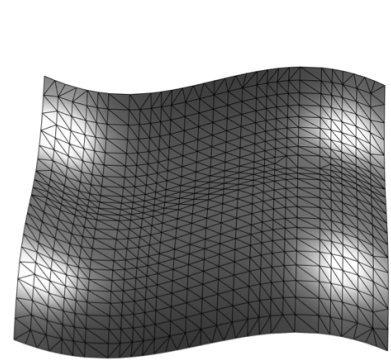

(a) Surface representation

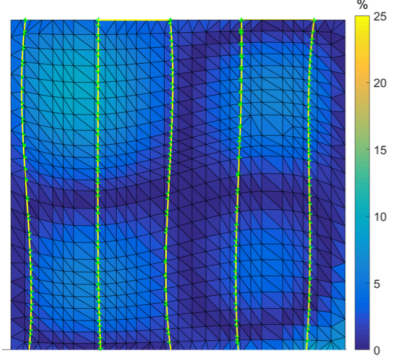

(b) Evolution of LSCM error (c) $\overline{e_{L S C M}}=3.14 \%$

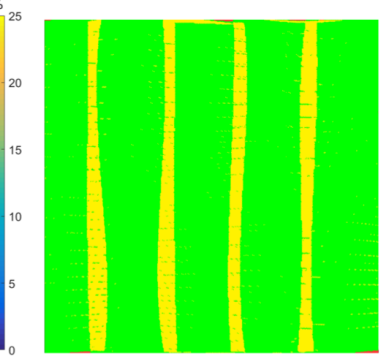

(Yellow)

Fig. 19 LSCM error for a high-curvature variation surface

\section{Conclusion}

In this paper, we have proposed a new method of scan path planning, ISOvScan, for automated inspection of manufactured part using an industrial robot. The originality of the method is the control of the overlap between two successive scanning passes. Actually, a scan path with the control of the overlap can control the orientation and the coverage rate of the laser beam. Therefore, digitizing quality, as well as digitizing time, can be controlled by optimizing overlapping zones. The method relies on the use of the Least-Square Conformal Map to transform the 3D surface into a $2 \mathrm{D}$ space. Therefore, scan path calculation can be performed more simply in the 2D space. The benefits of the ISOvScan method was assessed by results obtained from a real experimentation using an industrial robot equipped with a laser-scanner Zephyr. Nevertheless, the method has two main limitations. The first one concerns high-curvature variation surfaces for which the control of overlap turns out to be difficult. The second one is related to the way the driven points are calculated in the $2 \mathrm{D}$ space. As driven points are generated from the intersection between the equidistant planes and the facets, they are not regularly spaced, which may cause sudden changes of scanner orientation when the scanner moves from one point to another, and lead to undesirable noise in some areas on the surface. To attempt to solve this issue, future work will focus on the scan path smoothing using B-spline approximation. The scan path as a polynomial curve is actually better adapted to the robot motion control.

\section{Acknowledgements}

This research was made possible by the equipment support from Kreon Technology. We gratefully acknowledge the help provided by Mr. Tom Ranger and Mr. Dorian Verdel for their technical assistance in our experimental work.

\section{References}

1. S. Larsson and Johan AP Kjellander, Path planning for laser scanning with an industrial robot, Robotics and Autonomous Systems, 56(7), pp. 615-624 (2008) 
2. A. Bernard, M. Véron, Visibility theory applied to automatic control of 3D complex parts using plane laser sensors, CIRP Annals-Manufacturing Technology, 49(1), pp. 113-118 (2000)

3. F. Xi and C. Shu, CAD-based path planning for 3-D line laser scanning, Computer-Aided Design, 31(7), pp. 473-479 (1999)

4. H. ElMaraghy and X. Yang, Computer-aided planning of laser scanning of complex geometries, CIRP Annals-Manufacturing Technology, 52(1), pp. 411-414, (2003)

5. W. Derigent, E. Chapotot, G. Ris, S. Remy, and A. Bernard, 3D digitizing strategy planning approach based on a CAD model, Journal of Computing Information Science and Engineering,7(1), pp. 10-19 (2007)

6. K. Lee and H. Park. Automated inspection planning of free-form shape parts by laser scanning, Robotics and Computer-Integrated Manufacturing, 16(4):pp. 201-210, (2000)

7. F. Prieto, H. Redarce, R. Lepage, and P. Boulanger, Range image accuracy improvement by acquisition planning. In Proceedings of the 12th conference on vision interface (VI99), Trois Rivieres, Québec, Canada, pp.18-21 (1999)

8. Y. Quinsat and L. Sabourin, Optimal selection of machining direction for three-axis milling of sculptured parts. International Journal of Advanced Manufacturing Technologie, 27(112),pp. 11321139 (2006)

9. M. Mahmud, D. Joannic, M. Roy, A. Isheil, and J.-F. Fontaine, 3D part inspection path planning of a laser scanner with control on the uncertainty, Computer-Aided Design, 43(4), pp. 345-355 (2011)

10. C. Yang and F. Ciarallo, Optimized sensor placement for active visual inspection, Journal of Robotic Systems, 18(1), pp. 1-15 (2001)

11. C. Lartigue, Y. Quinsat, C. Mehdi-Souzani, A. Zuquete-Guaratoand S. Tabibian, Voxelbased path planning for 3D scanning of mechanical parts, Computer-Aided Design and Applications, 11(2), pp. 220-227 (2014)

12. R. Martins, G. Garca-Bermejo, Z. Casanova, R. Peran Gonzalez, Automated 3D surface scanning based on CAD model, Mechatronics, 15, pp. 837-857 (2005)

13. S. Son, H. Park, and K. Lee, Automated laser scanning system for reverse engineering and inspection, International Journal of Machine Tools and Manufacture, 42(8), pp. 889-897 (2002)

14. A. Mavrinac, X. Chen, and J. Alarcon-Herrera, Semiautomatic model-based view planning for active triangulation 3-D inspection systems. IEEE/ASME Transactions on Mechatronics, 20(2), pp. 799-811 (2015)

15. R. Raffaeli, M. Mengoni, M. Germani, Context dependent automatic view planning: the inspection of mechanical components, Computer-Aided Design and Applications, 10(1), pp. 111-127 (2013)

16. Q. Wu, J. Lu, W. Zou, and D. Xu, Path planning for surface inspection on a robotbased scanning system, IEEE International Conference on Mechatronics and Automation (ICMA), pp. 2284-2289 (2015)

17. T. Koutecky, D. Palousek, and J. Brandejs, Sensor planning system for fringe projection scanning of sheet metal parts, Measurement, 94, pp. 60-70 (2016)

18. C. Mineo, S. G. Pierce, P. I. Nicholson, and I. Cooper, Robotic path planning for non-destructive testing-A custom MATLAB toolbox approach, Robot. Comput. Integr. Manuf., 37, pp. 1-12 (2016)

19. M. Andulkar, S. Chiddarwar, and A. Marathe, Novel integrated offline trajectory generation approach for robot assisted spray painting operation, Journal of Manufacturing Systems, 37, pp. 201-216 (2015)

20. P. Atkar, D. Conner, A. Greenfield, H. Choset, and A. Rizzi, Uniform coverage of simple surfaces embedded in R3 for auto-body painting, Workshop on algorithmic foundations of robotics, pp. 383-398 (2004)

21. C. Tournier and E. Duc, A surface based approach for constant scallop height tool-path generation, International Journal of Advanced Manufacturing Technology, 19(5), pp. 318$324(2002)$

22. A. Can and A. Unuvar, A novel iso-scallop tool-path generation for efficient five-axis machining of free-form surfaces, International Journal of Advanced Manufacturing Technology, 51(912), pp. 1083-1098, (2010)

23. W. Li, Z. Yin, Y. Huang, T. Wu, and Y. Xiong, Tool path generation for triangular meshes using least-squares conformal map, International Journal of Production Research, 49(12), pp. 3653-3667 (2011) 
24. J. Zhao, Q. Zou, L. Li, and B. Zhou, Tool path planning based on conformal parameterization for meshes, Chinese Journal of Aeronautics, 28(5), pp. 1555-1563 (2015)

25. S. Haker, S. Angenent, A. R. Tannerai, R. Kikinis, G. Sapiro, and M. Halle, Conformal surface parameterization for texture mapping, IEEE Transactions on Visualization and Computer Graphics, 6(2), pp. 181-189 (2000)

26. B. Lévy, S. Petitjean, N. Ray, and J. Maillot, Least squares conformal maps for automatic texture atlas generation, ACM Trans. Graph., 21(3), pp. 362-371 (2002)

27. K. Hormann, B. Lévy, and A. Sheffer, Mesh parameterization: Theory and practice, ACM SIGGRAPPH, course notes, (2007)

28. C. Mehdi-Souzani, Y. Quinsat, C. Lartigue, and P. Bourdet, A knowledge database of qualified digitizing systems for the selection of the best system according to the application, CIRP Journal of Manufacturing Science and Technology., 13, pp. 15-23 (2016)

29. M. Phan, Y. Quinsat, C. Lartigue, Simulation of Laser-Sensor Digitizing for On-Machine Part Inspection, Advances on Mechanics, Design Engineering and Manufacturing, Part of the series Lecture Notes in Mechanical Engineering pp. 301-311 (2016) 